\title{
SPECT imaging of glioma with radioiodinated CLINDE: evidence from a mouse GL26 glioma model
}

Stergios Tsartsalis ${ }^{1,2}$, Noé Dumas $^{1 \dagger}$, Benjamin B Tournier ${ }^{1 \dagger}$, Tien Pham $^{3}$, Marcelle Moulin-Sallanon ${ }^{4}$, Marie-Claude Grégoire ${ }^{3}$, Yves Charnay ${ }^{1}$ and Philippe Millet ${ }^{1 *}$

\begin{abstract}
Background: Recent research has demonstrated the potential of 18-kDa translocator protein (TSPO) to serve as a target for nuclear imaging of gliomas. The aim of this study was to evaluate SPECT imaging of GL26 mouse glioma using radioiodinated CLINDE, a TSPO-specific tracer.

Methods: GL26 cells, previously transfected with an enhanced green fluorescent protein (EGFP)-expressing lentivirus, were stereotactically implanted in the striatum of C57/Bl6 mice. At 4 weeks post-injection, dynamic SPECT scans with $\left[{ }^{123} \mid\right] C$ CINDE were performed. A displacement study assessed specificity of tracer binding. SPECT images were compared to results of autoradiography, fluorescence microscopy, in situ nucleic acid hybridization, histology, and immunohistochemistry. Western blotting was performed to verify TSPO production by the tumor.

Results: Specific uptake of tracer by the tumor is observed with a high signal-to-noise ratio. Tracer uptake by the tumor is indeed $3.26 \pm 0.32$ times higher than that of the contralateral striatum, and $78 \%$ of the activity is displaceable by unlabeled CLINDE. Finally, TSPO is abundantly expressed by the GL26 cells.

Conclusions: The present study demonstrates the feasibility of ${ }^{[23}$ I]CLINDE SPECT in translational studies and underlines its potential for clinical glioma SPECT imaging.
\end{abstract}

Keywords: TSPO; CLINDE; Glioma; SPECT

\section{Background}

The $18-\mathrm{kDa}$ translocator protein (TSPO) is primarily located at the contact sites between the inner and outer mitochondrial membranes, as a component of the mitochondrial permeability transition pore. Although its expression in healthy brain is minimal, it increases substantially in neuroinflammatory conditions [1] as well as in brain malignancies [2-4]. In the case of brain tumors, TSPO is predominantly expressed by neoplastic cells, thus, it is regarded as a potential central nervous system (CNS) cancer imaging biomarker.

Radioiodinated CLINDE is a highly-specific SPECT TSPO tracer that has been already evaluated in animal

\footnotetext{
* Correspondence: philippe.millet@hcuge.ch

${ }^{\dagger}$ Equal contributors

${ }^{1}$ Vulnerability Biomarkers Unit, Division of General Psychiatry, Department of Mental Health and Psychiatry, University Hospitals of Geneva, Chemin du Petit-Bel-Air 2, CH1225 Geneva, Chêne-Bourg, Switzerland

Full list of author information is available at the end of the article
}

models of neuroinflammation [5,6]. The aim of this study was to investigate the potential use of radioiodinated CLINDE as a brain tumor SPECT imaging tracer in the mouse GL26 glioma model using in vivo highresolution SPECT [7]. To validate our approach, we 1) compared in vivo measurements to ex vivo autoradiography and histology; 2) evaluated the specificity of tracer binding (in vivo displacement procedure); and 3) confirmed the presence of TSPO mRNA by means of in situ hybridization, as well as TSPO protein levels produced by the tumor by means of immunohistochemistry and western blotting, respectively.

\section{Methods}

All chemicals were purchased from Sigma-Aldrich (St. Gallen, Switzerland), unless otherwise specified. CLINDE precursor was provided by the Australian Nuclear Science and Technology Organization (ANSTO). 
GL26 mouse glioma cells were kindly provided by Prof. L. Zitvogel (Institut G. Roussy, Paris, France). They were routinely cultured in DMEM, supplemented with $10 \%$ fetal bovine serum (FBS) and $1 \mathrm{mM}$ sodium pyruvate (Life technologies, Zug, Switzerland). A stable GL26 cell line expressing enhanced green fluorescent protein (EGFP) was obtained by a lentiviral vector transfection according to the manufacturer instructions (ViraPower Lentiviral Expression System, Life technologies, Zug, Switzerland). Blasticidin (from 2 to $5 \mu \mathrm{g} / \mathrm{ml}$ of culture medium during 2 weeks) was used for the selection of clone of GL26 cells expressing EGFP. GL26-EGFP cells (50.000 cells in $2.5 \mu \mathrm{l}$ of culture medium/injection) were stereotactically implanted in the striatum of adult male C57/Bl6 mice (Janvier Laboratories, Saint Berthevin, France, $n=10$ ), weighing $28.2 \pm 3.2 \mathrm{~g}$, under isoflurane ( $4 \%$ induction and $1.5 \%$ to $2 \%$ maintenance) anesthesia. Three of these animals received a concurrent second injection of culture medium only in the contralateral striatum. All animal experiments were approved by the Animal Ethics Committee of the Canton of Geneva and were in accordance with the European Union regulations on animal research.

$\left[{ }^{123} \mathrm{I}\right]$ CLINDE were labeled as previously described [8]. At 4 weeks post-injection, mice were injected with 62.24 $\pm 18.90 \mathrm{MBq}$ of radiotracer and underwent a dynamic SPECT session with the U-SPECT-II scanner (MILABS, Utrecht, Netherlands), using a list mode acquisition protocol consisting of 60 frames of $84 \mathrm{~s}$. For the displacement study, four out of ten mice were injected with unlabeled CLINDE $(10 \mathrm{mg} / \mathrm{kg})$ at the 82 th minute post-injection of the labeled tracer. Total scan duration was $165 \mathrm{~min}$.

SPECT tomograms were reconstructed with an ordered subsets expectation maximization (OSEM) algorithm (using four subsets and six iterations). SPECT data were corrected for radioactive decay, but no correction of attenuation and scatter was applied. A 0.4-mm FWHM filter was applied on images. The dynamic SPECT images were first averaged over time frames between 50 and $80 \mathrm{~min}$ in order to enhance visualization of the different structures. These images were manually co-registered to a mouse magnetic resonance imaging (MRI) and volume of interest (VOI) template [9] that was implemented in PMOD software (version 3.6, 2014, PMOD Technologies Ltd, Zurich, Switzerland), and the co-registration parameters were employed for dynamic image co-registration. Standardized uptake values (SUV) of tumor and contralateral striatum radioactivity were extracted from averaged frames between 50 and $80 \mathrm{~min}$, using PMOD and compared by means of $t$-test (Microsoft Excel, 14.4.7).

Mice were sacrificed after the end of the scan session at 4 weeks post-implantation of GL26 cells. Brains were harvested and frozen in isopentane $\left(-20^{\circ} \mathrm{C}\right)$ and serial coronal sections were taken. Brain sections were used for ex vivo autoradiography, fluorescence microscopy, Nissl staining, immunohistochemistry, and in situ hybridization. Two digoxigenin-labeled riboprobes complementary to the Mus musculus translocator protein (TSPO) mRNA sequences (NM-00977.4: coding sequences 102-380 and 322-577, respectively) were used. The cDNA amplicons from total RNA extracted from a mouse brain cortex were inserted in a TOPO-pCR4 vector (Invitrogen, Carlsbad, CA, USA) followed by linearization and in vitro transcription in presence of dUTP-digoxigenin (Roche, Basel, Switzerland) according to the manufacturer instructions (Invitrogen, Carlsbad, CA, USA). The in situ hybridization histochemistry procedures were previously reported in detail [10]. The use of sense probes, in serial tissue sections, resulted in the absence of any hybridizing signal whereas the two antisense independent probes mentioned above gave the same patterns of labeling. Thus, these data were in favor of the specificity of the hybridizing signal for TSPO mRNA visualized in the tumor tissue sections. TSPOimmunoreactivity expressed by glioma cells was further suggested by immunohistochemistry and the TSPOimmunoreactive band seen in a Western blot using an anti-TSPO antibody (goat anti-mouse TSPO antibody LS-B5755, LifeSpan Biosciences, Inc., Seattle, WA, USA; working dilution 1:1,000). The Western blotting procedure was published with detail elsewhere [11].

\section{Results}

Figure $1 \mathrm{~A}, \mathrm{~B}, \mathrm{C}$ shows an in vivo SPECT image (averaged over frames corresponding to 50 to $80 \mathrm{~min}$ post-injection of tracer), co-registered to the mouse MRI template. A corresponding coronal brain section after ex vivo autoradiography is presented in Figure 1D. Figure 2 (left part) illustrates the tissue-activity curves (TACs) extracted from the dynamic images of seven mice using two circular VOIs manually delineated on the tumor as well as an equal size VOI on the contralateral striatum. A rapid tracer uptake is observed in the initial frames post-injection followed by washout that, for the contralateral VOI, is almost complete and rapid. Analysis of averaged image frames between 50 and 80 min post-injection across different experimental subjects demonstrated that the tumor side (mean SUV 1.04 \pm 0.22 ) presents a level of radioactivity $3.26 \pm 0.32$ times higher than that of the contralateral side (mean SUV $0.32 \pm 0.08, p<0.001$ ). TACs of the dynamic scan presented in Figure 2 (right part) also depict the result of a displacement experiment in which $10 \mathrm{mg} / \mathrm{kg}$ of unlabeled compound were injected at $82 \mathrm{~min}$ postinjection. Radioactivity kinetic pattern in the tumor VOI reveals that about $78 \%$ of the radioactivity is displaceable.

Figure $3 \mathrm{~A}$ presents a coronal section of a tumor-bearing mouse after Nissl staining while Figure 3B depicts brain 


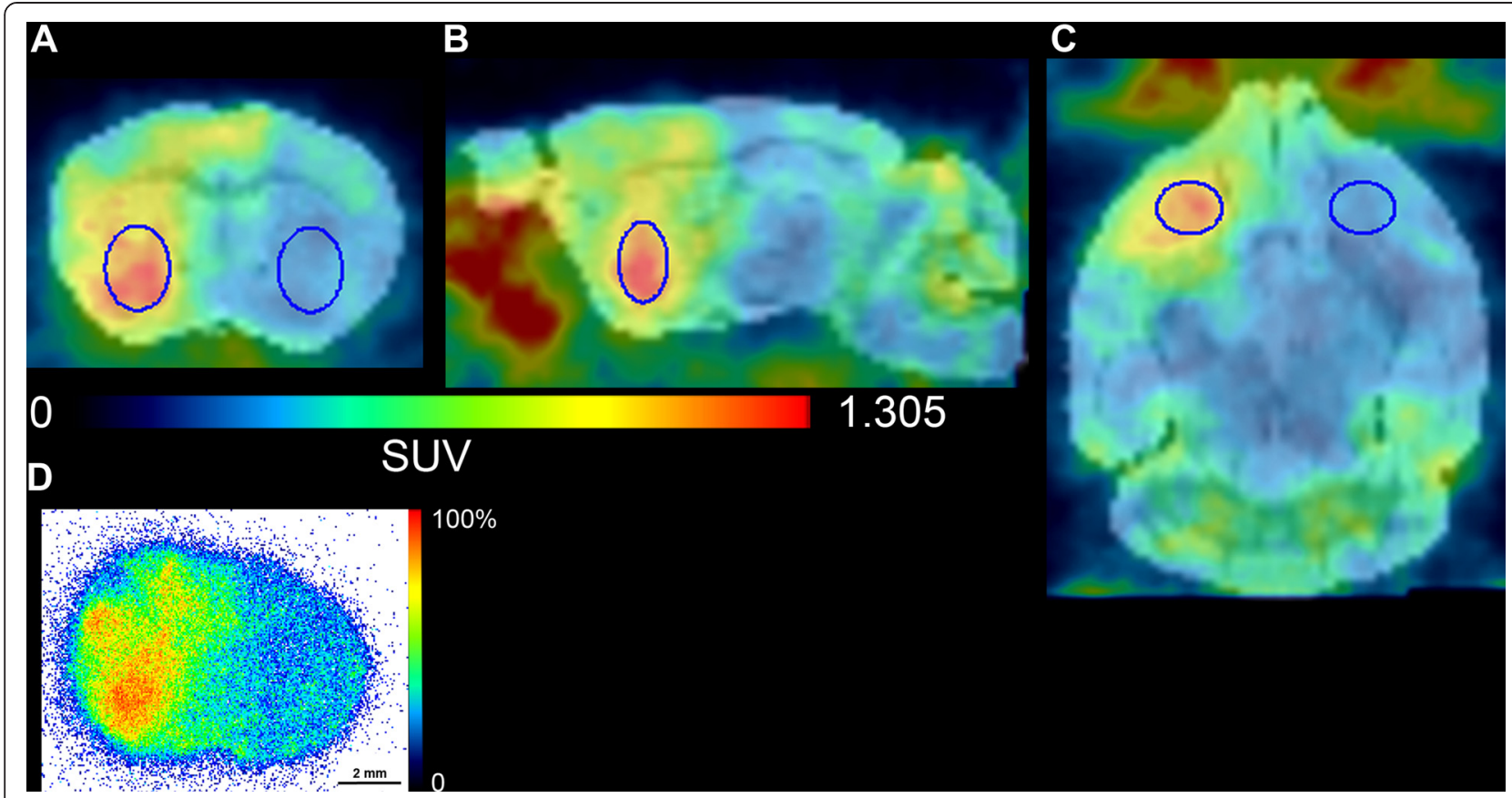

Figure 1 In vivo SPECT imaging, histology and ex vivo autoradiography. In vivo SPECT image (summed frames between 50 and 80 min of scan), co-registered with a mouse brain MRI template, obtained from one mouse bearing the GL26 tumor in the coronal (A), sagittal (B), and axial (C) planes. VOls corresponding to tumor and contralateral brain tissue are also depicted. (D) Ex vivo autoradiography of a corresponding brain section from the same mouse. Color scale refers to percentage of maximal activity in the image.

sections from another mouse that was analyzed by means of fluorescence microscopy and (Figure 3C) in situ hybridization with specific probes that demonstrate the expression of the TSPO mRNA by the tumor.

Finally, immunohistochemical analysis (Figure 4A) shows TSPO-immunoreactivity in most of the tumor cells. Western blotting (Figure 4B) analyses of tumor tissue extracts compared to spleen extracts (as positive control) show a similar $18-\mathrm{kDa}$ band confirming the presence of TSPO protein in these cell populations.

\section{Discussion}

Standard clinical practice in brain tumor imaging includes MRI that is considered the 'gold standard' presenting high spatial resolution but failing to provide sufficient information on the histopathological and molecular profile of the malignant lesions [12]. On the contrary, nuclear imaging approaches such as positron emission tomography (PET) and SPECT may provide information on functional features of the disease such as metabolic activity and the expression of molecules

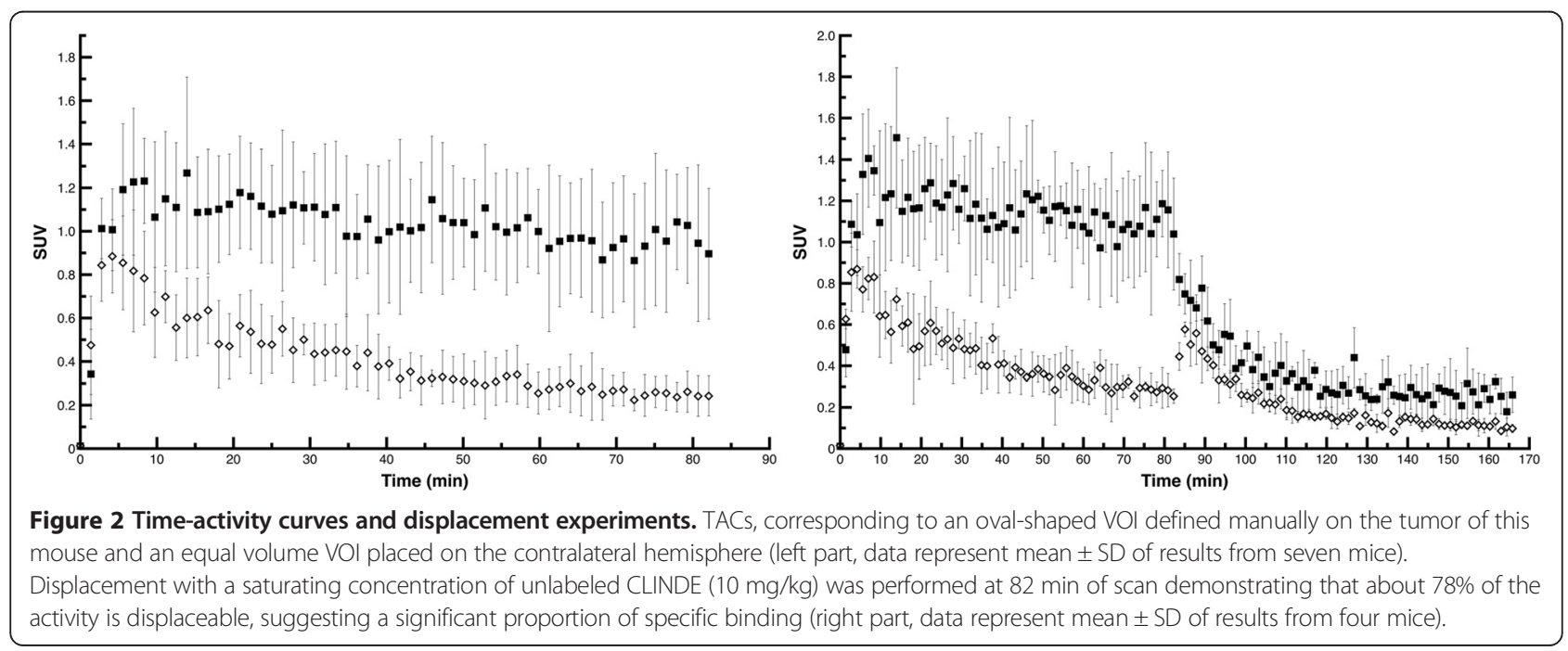




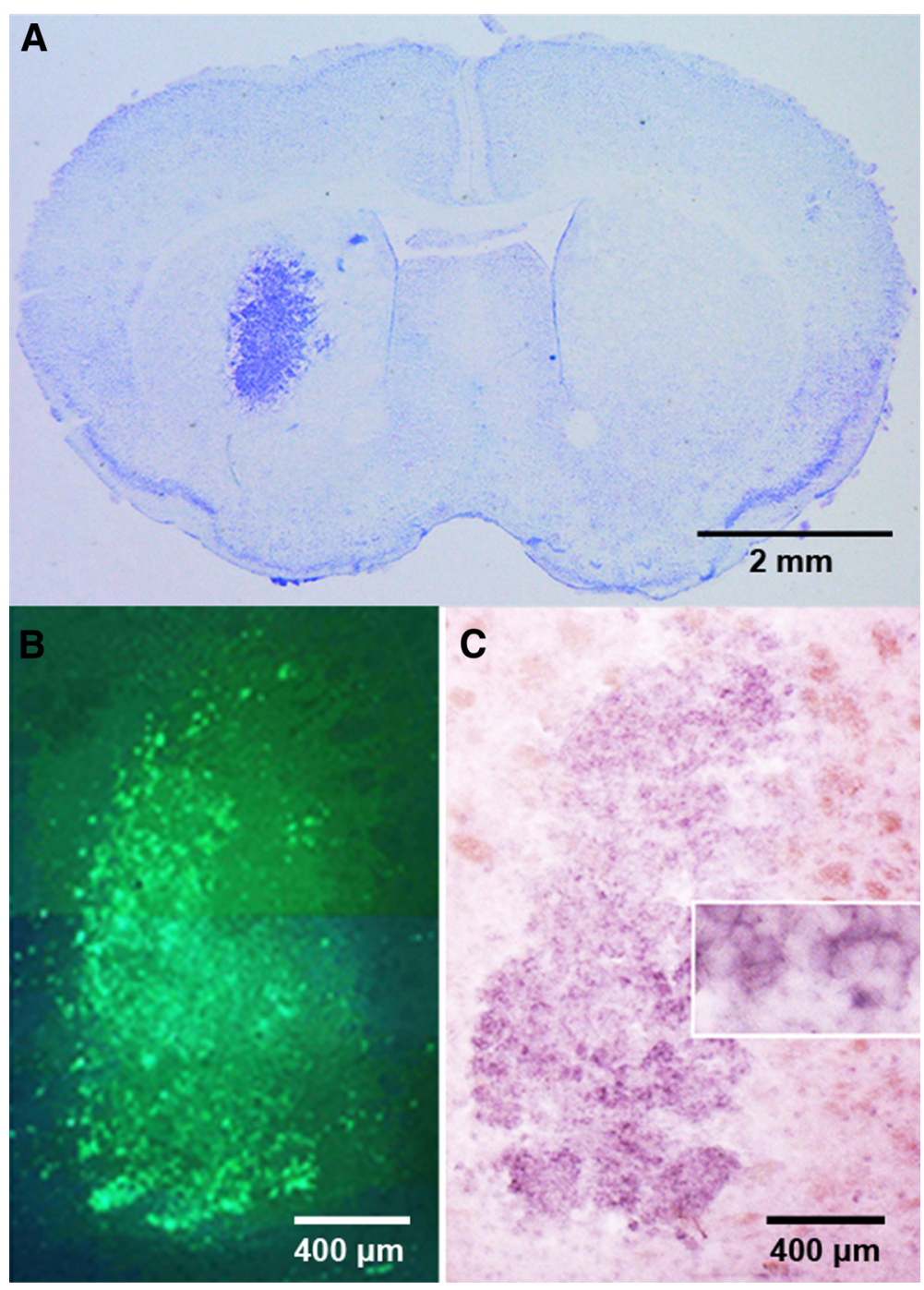

Figure 3 Fluorescence microscopy and in situ mRNA hybridization. (A) Coronal brain section of GL-26 tumor-bearing mouse visualized after Nissl staining. (B) A coronal brain section of another mouse visualized under fluorescence microscopy depicting the EGFP-expressing GL26 cells. (C) Result of in situ TSPO mRNA hybridization performed on an adjacent brain section (box depicts part of the slice in a greater magnification).
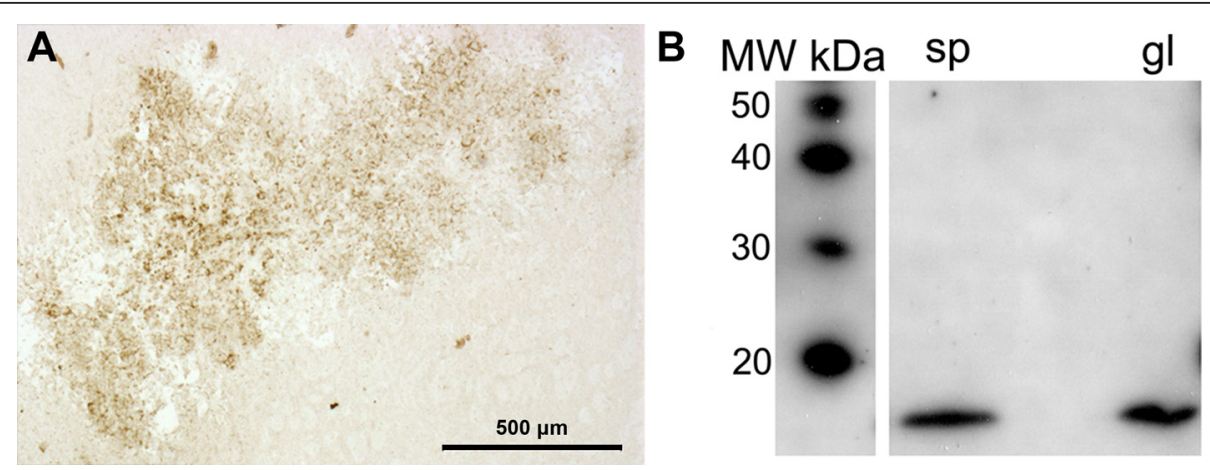

Figure 4 Immunohistochemistry and Western blotting. (A) Immunohistochemical analysis of a brain section from a mouse showing TSPO expression by tumor cells. (B) Western blotting assay demonstrates TSPO expression from GL26 tumor tissue (gl) as compared to spleen tissue (sp), used as a positive control. Molecular weight of the bands corresponds to $18 \mathrm{kDa}$, as demonstrated with the use of a molecular weight probe (left-most column). 
specifically associated with this type of pathological processes of the CNS, one of them being TSPO. There is now evidence that TSPO expression might serve as a biomarker of tumor progression, metastatic potential, and overall prognosis in several human tumors [13].

TSPO imaging in glioma has already been assessed using PET through a series of studies using different $\left[{ }^{11} \mathrm{C}\right]$ - or $\left[{ }^{18} \mathrm{~F}\right]$-labeled tracers $[2-4,14]$. Buck et al. [14] showed specific $\left[{ }^{18} \mathrm{~F}\right]$ PBR06 uptake that closely correlated with TSPO expression in a rat glioma model. Similarly, [ ${ }^{18}$ F]DPA-714 preclinical imaging in rat models gave encouraging results [2,3]. Winkeler et al. [2] showed that neoplastic cells produce the majority of TSPO expressed by the tumor. In this study, TSPO expression by GL26 cells, as assessed by means of in situ hybridization for TSPO mRNA and fluorescence microscopy, supports the aforementioned observation.

Our study highlights the potential of SPECT imaging in clinical neuro-oncology. In fact, SPECT is frequently employed in clinical neuroscience: in the domain of neurooncology, recent meta-analyses of clinical studies demonstrate that SPECT, due to its high specificity, is perhaps the best imaging modality to differentiate between cerebral regions due to tumor recurrence or radiotherapy-induced necrosis $[15,16]$. Regarding TSPO imaging in particular, Awde et al. proposed that TSPO imaging could serve as a molecular biomarker of glioma progression and even response to treatment [17]. However, regarding human glioma imaging using TSPO-specific radiotracers, one has to take into account that not all types of glioma show equally high levels of TSPO expression as glioblastoma $[4,18]$ and this element could be exploited for the differential diagnosis of different types of these tumors. In addition, fundamental differences between SPECT and PET render the former much more readily available in clinical settings due to the long half-lives of radionuclides employed in SPECT (e.g., $13.2 \mathrm{~h}$ for ${ }^{123} \mathrm{I}$ compared to $110 \mathrm{~min}$ for ${ }^{18} \mathrm{~F}$ and 20 min for ${ }^{11} \mathrm{C}$, two widely employed PET radionuclides) and its lower cost. Similarly, in the experimental level, the emergence of new preclinical SPECT scanners provides sub-millimeter spatial resolution, superior to that of small animal PET and has rendered SPECT a particularly powerful tool for small animal research [19]. This, along with the aforementioned advantages of SPECT in the practical level (availability of radionuclides, lower cost), propose that employing radioiodinated CLINDE could be considered an attractive alternative to PET TSPO tracers already described above, in experimental and - potentially - in clinical settings. Indeed, in the present study, we demonstrate a specific binding of radioiodinated CLINDE to GL26 glioma, where TSPO expression is abundant. Tracer uptake by the tumor is 3.26 times higher than in the contralateral hemisphere, indicating a good signal-to-noise ratio. In vivo SPECT images, ex vivo autoradiography, and fluorescence microscopy show comparable results regarding tumor anatomy. A displacement study confirmed the specificity of tracer binding on TSPO as about $78 \%$ of the activity in the tumor was displaceable by the unlabeled compound. In Figure 2 (right part), an augmentation of the activity in the striatum that is contralateral to the one with the tumor after displacement with CLINDE is observed. It could be attributed to a displacement of the radiotracer from extracerebral tissues where TSPO expression is abundant [1] and entry into the brain. Finally, tracer uptake from tumor is not attributable to the lesion produced by the stereotactic injection of GL26 cells, as contralateral striatum, injected with culture medium alone, did not reveal but a negligible uptake (data not shown).

\section{Conclusions}

To our knowledge, this is the first study on a potential TSPO SPECT glioma tracer. We believe that, given its specificity and its signal-to-noise ratio, our results demonstrate the quality of $\left[{ }^{123} \mathrm{I}\right]$ CLINDE SPECT imaging of glioma in translational studies and are indicative of its potential value in a clinical setting.

\section{Competing interests}

The authors declare that they have no competing interests.

\section{Authors' contributions}

ST, YC, and PM conceived and designed the study. ST, ND, BBT, TP, MM, and PM participated in data acquisition and interpretation. ST, BBT, and YC drafted the manuscript and MG, YC, and PM revised it. All authors read and approved the final form of the manuscript.

\section{Acknowledgements}

The authors wish to thank the Australian Nuclear Science and Technology Organization (ANSTO) for providing the CLINDE precursor, Pr. L. Zitvogel (Institut G. Roussy, Paris, France) for providing the GL26 cells. In addition, Pr. Laurent Roux and Dr Dominique Garcin of the University Medical Center of Geneva University for providing their expertise and the necessary infrastructure for lentivirus-related experiments. Finally, M. Surini-Demiri and M. Ben Ammar for their technical assistance. The authors are grateful for the contribution of the 'Association IFRAD Suisse,' created in 2009 at the initiative of the 'Fondation pour la Recherche sur Alzheimer' (formerly IFRAD France) and the Geneva Neuroscience Center.

\section{Author details}

'Vulnerability Biomarkers Unit, Division of General Psychiatry, Department of Mental Health and Psychiatry, University Hospitals of Geneva, Chemin du Petit-Bel-Air 2, CH1225 Geneva, Chêne-Bourg, Switzerland. 'Department of Psychiatry, University of Geneva, 1 rue Michel-Servet, CH1211 Geneva 4, Switzerland. ${ }^{3}$ ANSTO LifeSciences, Australian Nuclear Science and Technology Organisation, New Illawarra Road, Sydney, NSW 2234, Australia. ${ }^{4}$ INSERM, J. Fourier University, INSERM Unit 1039, Domaine de la Merci, 38700 La Tronche, France.

Received: 19 September 2014 Accepted: 24 February 2015 Published online: 13 March 2015

\section{References}

1. Scarf AM, Kassiou M. The translocator protein. J Nucl Med. 2011;52(5):677-80.

2. Winkeler A, Boisgard R, Awde AR, Dubois A, Theze B, Zheng J, et al. The translocator protein ligand [(1)(8)F]DPA-714 images glioma and activated microglia in vivo. Eur J Nucl Med Mol Imaging. 2012;39(5):811-23. 
3. Tang D, Hight MR, McKinley ET, Fu A, Buck JR, Smith RA, et al. Quantitative preclinical imaging of TSPO expression in glioma using N, N-diethyl-2-(2-(4(2-18 F-fluoroethoxy)phenyl)-5,7-dimethylpyrazolo[1,5-a]pyrimi din-3-yl) acetamide. J Nucl Med. 2012;53(2):287-94.

4. Su Z, Herholz K, Gerhard A, Roncaroli F, Du Plessis D, Jackson A, et al. [(1)(1) Cl-(R)PK11195 tracer kinetics in the brain of glioma patients and a comparison of two referencing approaches. Eur J Nucl Med Mol Imaging. 2013;40(9):1406-19.

5. Mattner F, Bandin DL, Staykova M, Berghofer P, Gregoire MC, Ballantyne P, et al. Evaluation of [(1)(2)(3)I]-CLINDE as a potent SPECT radiotracer to assess the degree of astroglia activation in cuprizone-induced neuroinflammation. Eur J Nucl Med Mol Imaging. 2011;38(8):1516-28.

6. Mattner F, Mardon K, Katsifis A. Pharmacological evaluation of [123I]-CLINDE: a radioiodinated imidazopyridine-3-acetamide for the study of peripheral benzodiazepine binding sites (PBBS). Eur J Nucl Med Mol Imaging. 2008;35 (4):779-89.

7. U-SPECT System. http://www.milabs.com/. Accessed 10 October 2012.

8. Mattner F, Katsifis A, Staykova M, Ballantyne P, Willenborg DO. Evaluation of a radiolabelled peripheral benzodiazepine receptor ligand in the central nervous system inflammation of experimental autoimmune encephalomyelitis: a possible probe for imaging multiple sclerosis. Eur J Nucl Med Mol Imaging. 2005;32(5):557-63.

9. Schiffer WK, Mirrione MM, Biegon A, Alexoff DL, Patel V, Dewey SL. Serial microPET measures of the metabolic reaction to a microdialysis probe implant. J Neurosci Methods. 2006;155(2):272-84.

10. Charnay Y, Imhof A, Vallet PG, Hakkoum D, Lathuiliere A, Poku N, et al. Clusterin expression during fetal and postnatal CNS development in mouse. Neuroscience. 2008;155(3):714-24.

11. Imhof A, Charnay Y, Vallet PG, Aronow B, Kovari E, French LE, et al. Sustained astrocytic clusterin expression improves remodeling after brain ischemia. Neurobiol Dis. 2006;22(2):274-83.

12. Chen W. Clinical applications of PET in brain tumors. J Nucl Med. 2007;48 (9):1468-81.

13. Batarseh A, Papadopoulos V. Regulation of translocator protein $18 \mathrm{kDa}$ (TSPO) expression in health and disease states. Mol Cell Endocrinol. 2010;327(1-2):1-12.

14. Buck JR, McKinley ET, Hight MR, Fu A, Tang D, Smith RA, et al. Quantitative, preclinical PET of translocator protein expression in glioma using 18 F-N-fluoroacetyl-N-(2,5-dimethoxybenzyl)-2-phenoxyaniline. J Nucl Med. 2011;52(1):107-14.

15. Shah AH, Snelling B, Bregy A, Patel PR, Tememe D, Bhatia R, et al. Discriminating radiation necrosis from tumor progression in gliomas: a systematic review what is the best imaging modality? I Neurooncol. 2013;112(2):141-52.

16. Zhang H, Ma L, Wu C, Xu B. Performance of SPECT in the differential diagnosis of glioma recurrence from radiation necrosis. J Clin Neurosci. 2015;22(2):229-37.

17. Awde AR, Boisgard R, Theze B, Dubois A, Zheng J, Dolle F, et al. The translocator protein radioligand 18 F-DPA-714 monitors antitumor effect of erufosine in a rat $9 \mathrm{~L}$ intracranial glioma model. J Nucl Med. 2013;54 (12):2125-31.

18. Su Z, Gerhard A, Hinz R, Coope DJ, Thompson G, Karabatsou K, et al. In vivo imaging of translocator protein expression in low-grade gliomas by positron emission tomography. Glasgow, UK: British Neuro-Oncology Society Annual Conference; 2010.

19. Meikle SR, Kench P, Kassiou M, Banati RB. Small animal SPECT and its place in the matrix of molecular imaging technologies. Phys Med Biol. 2005;50 (22):R45-61.

\section{Submit your manuscript to a SpringerOpen ${ }^{\odot}$ journal and benefit from:}

- Convenient online submission

Rigorous peer review

- Immediate publication on acceptance

- Open access: articles freely available online

- High visibility within the field

- Retaining the copyright to your article

Submit your next manuscript at $\gg$ springeropen.com 